\title{
Could Ambulatory Blood Pressure Monitoring Be a Routine Investigation for Patients with Mild Cognitive Impairment?
}

\author{
Mohamed Hamdy Ibrahim1, Tamer Taha Ismail Taha², Laila Adel Mohsen³, \\ Nisrin Moustafa Rizk El-Elsaadouni ${ }^{4}$ \\ ${ }^{1}$ Neurology Department, Faculty of Medicine, Ain Shams University, Cairo, Egypt \\ ${ }^{2}$ Cardiology Department, Faculty of Medicine, Minia University, Minya, Egypt \\ ${ }^{3}$ Radiology Department, Faculty of Medicine, Minia University, Minya, Egypt \\ ${ }^{4}$ Psychiatry Department, Faculty of Medicine, Al-Mansoura University, Mansoura, Egypt \\ Email: mohamedhamdy neuro2007@yahoo.com
}

Received 28 March 2015; accepted 9 May 2015; published 15 May 2015

Copyright (C) 2015 by authors and Scientific Research Publishing Inc.

This work is licensed under the Creative Commons Attribution International License (CC BY). http://creativecommons.org/licenses/by/4.0/

(c) (i) Open Access

\begin{abstract}
Objectives: To study the ambulatory measured blood pressure (ABPM) profile in normotensive patients with mild cognitive impairment (MCI). Patients and Methods: The study was designed as a case control study including 50male patients with mild cognitive impairment in the age group of 30 - 50 years old. The control group included 30 volunteers with no cognitive impairment and in the same age group (30 - 50 years old) and same gender. Mini-mental estate examination, office and ABP monitoring (ABPM) and brain MRI scans were done for cases and controls. Results: Thirty patients (60\%) with MCI revealed a non-dipper blood pressure pattern. Sleeping systolic blood pressure and sleeping systolic load were significantly higher in patients with MCI than in normal volunteers $(p=0.01)$. MRI brain showed more white matter lesions (WMLs) in patients with MCI than in normal volunteers; however, this didn't reach significance level $(p=0.056)$. Conclusion: MCI in normotensive young adult patients could reflect an abnormal circadian blood pressure rhythm. Ambulatory blood pressure monitoring could be an essential investigation in young adult MCI patients.
\end{abstract}

\section{Keywords}

Mild Cognitive Impairment, Ambulatory Blood Pressure Monitoring, White Matter Lesions, Non-Dipper, MMSE 


\section{Introduction}

Mild cognitive impairment (MCI) refers to cognitive impairment that does not meet the criteria for dementia. Several criteria and subtypes of MCI have been proposed [1]-[3]. These criteria include a measurable deficit in cognition in at least one domain, in absence of dementia or impairment in activities of daily living. As originally constructed, the concept of MCI emphasizes memory impairment and is suggested to be a precursor state for Alzheimer disease (AD). Subsequently, it is recognized that MCI can be heterogeneous in terms of clinical presentation, etiology, prognosis, and prevalence [3]-[5]. Accordingly, the construct is expanded to broaden the scope of MCI to other cognitive domains, thereby extending the early detection of other dementias in their prodromal stages [6]-[8]. Cognitive function can be assessed by mini-mental state examination (MMSE) which is a brief 30 points questionnaire test. The test result may be classified as normal (score: 25 - 30), mild cognitive (score: 21 - 24) and severe cognitive impairment (score: 0 - 9).

Although the relationship between blood pressure and dementia risk is not clearly understood, most longitudinal studies have found that the risk of dementia and cognitive impairment is associated with either high blood pressure [9] [10] or with low blood pressure. However, other studies have found no association [11]-[13].

Ambulatory blood pressure monitoring (ABPM) is being increasingly recommended for routine clinical practice [1] [2]. It may be particularly useful in evaluating patients with variable blood pressure readings in the office, or patients with wide discrepancies between the blood pressure readings at home and at clinic (i.e. "white coat” hypertension). ABPM and, in particular, nocturnal blood pressure readings, may also provide prognostic data [3].

\section{Patients and Methods}

Fifty male patients were recruited from neurology and psychiatry clinics. These patients were diagnosed as having MCI in the form of mild decrease in short term memory span affecting their daily work without any associated comorbidity (i.e. psychiatric, vascular or metabolic disorders). These patients constituted Group 1. Thirty volunteers were recruited and were age and gender matched. These volunteers were either relatives of patients or health workers from the same hospital. They had a normal Mini-mental state examination (MMSE) score denoting absence of cognitive impairment, fulfilled the inclusion criteria for controls and had no evidence of the exclusion criteria mentioned later. These controls constituted Group 2.

\subsection{Inclusion Criteria}

Inclusion criteria for patients and controls included male gender, age range of 35 - 50 years old and normal average blood pressure on ABPM. Sphygmomanometer arterial blood pressure monitoring device has been used for office blood pressure monitoring for patients, the inclusion MMSE score is 21 - 24 which indicates MCI. For controls, the inclusion MMSE score is 25 - 30 which indicates a normal cognitive function.

\subsection{Exclusion Criteria}

Exclusion criteria include female gender, documented hypertension, associated co-morbidities (e.g. diabetes, psychiatric disorders, and metabolic disorders). MMSE scores lower than 21 were not included. Inability to perform a 24-hour ABPM or MMSE evaluation was also exclusion criteria in this study. Patients with missing data were also excluded.

\subsection{Neuropsychiatric Assessment}

Patients and controls that fulfilled the above criteria were subject to thorough clinical evaluation, including history taking and general examination. Neurological and psychiatric examinations were carried out to exclude any associated neurological or psychiatric disorders, e.g. pseudo-dementia with underlying depression. Mini-mental estate examination (MMSE) was then done to all patients and controls. The mini-mental state examination (MMSE) or Folstein test is a brief 30-point questionnaire test that is used to screen for cognitive impairment [4]. We used an Arabic version of the MMSE score [5].

\subsection{Cardiac Assessment and Blood Pressure Evaluation}

Cardiac examination was done next, including office blood pressure measurement. Office BP measurement was 
done as follows: Patient was allowed to rest for 5 minutes in supine position then blood pressure was recorded using a standard mercury sphygmomanometer in the supine position as well. Three clinic BP readings were taken on 3 separate occasions, at least 2 weeks apart. The reported clinic blood pressure reading is the average of the 3 recorded readings. Hypertension is considered if the average BP exceeded 140/90 mmHg in office [2]. In 24-hour ABPM, hypertension is considered if the average BP exceeded 130/80 $\mathrm{mmHg}$ [3]. As previously described, hypertensive patients were excluded from the study. Normotensive patients and controls were included in the study. The morning and nocturnal variations in blood pressure were evaluated and compared.

\subsection{MRI Examination}

The next day, patients and controls visited the radiology department for a customized simple brain MRI study that is composed of standard axial T1 weighted, axial T2 weighted and axial FLAIR sequences. The MRI study was done on a 0.2T GE Signa Excite machine (GE Medical Systems, Waukesha, WI). The evaluation of the MRI was to rule out other causes of cognitive impairment and to score the white matter hyperintensities (Figure 1). The deep white matter hyperintensities were evaluated and scored according to the modified Scheltens score [7].

Because we are dealing with patients in an under-developed country (many of whom are poorly educated), we used this simple MRI evaluation and score to confirm the degree of white matter hyperintensities and whether or not, they agree with our findings.

The MMSE and MRI scoring were each performed by a single experienced physician each with 10 years' experience in their fields. Similarly, the 24-hour ABPM analysis was done by an experienced cardiologist with 12 years' experience.

A blood sample was also withdrawn from all patients to be analyzed for their dyslipidemic profile and random blood sugar.

\subsection{Ethical Statement}

The study was approved by the institution's local ethics committee which conforms with the Declaration of Helsinki. The study objectives and design were explained to patients and controls. Inclusion into the study was done after patients gave written informed consent.

\subsection{Statistical Analysis}

Statistical analysis was carried out using SPSS version 21 for windows (SPSS Inc., Cary, NC). The analysis of the difference of nominal data, e.g. smoking and dyslipidemia, between groups was carried out using the Chi Square test. Student T-test was then used to compare the means of numerical data, e.g. age, blood pressure and

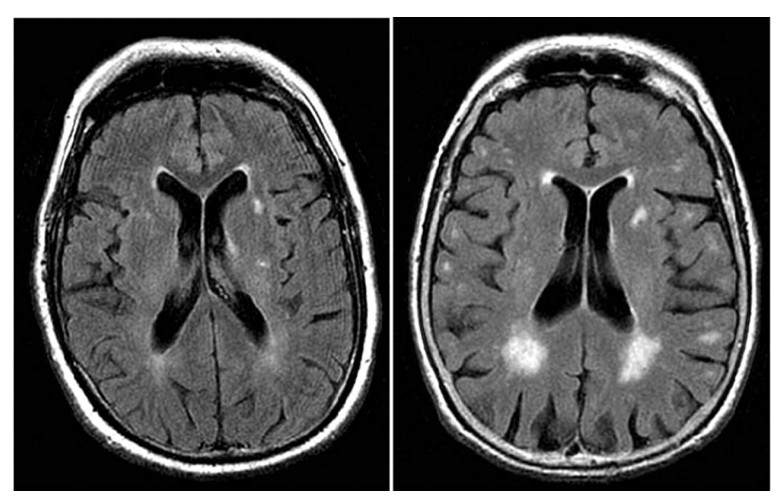

Figure 1. White matter hyperintensities on magnetic resonance imaging (axial fluid attenuated inversion recovery sequence) in two 40 year old patients: (left) minor white matter hyperintensities; (right) extensive white matter hyperintensities predominating in periventricular region. White matter lesions are considered present if hyperintense on T2 weighted, fluid attenuated inversion recovery, and proton density images, without prominent hypointensity on $\mathrm{T} 1$ weighted images. 
echocardiographic parameters between both groups. Mann Whitney U test was used for comparing the MRI score between both groups. Bivariate Spearman's correlations were carried out between each of the office BP measurements and the 24-hour ABPM variables and the MMSE and the MRI score. A linear regression analysis was run to find the factors with stronger impact on the cognitive function. Significance level for the used tests is $\mathrm{p}=0.05$.

\section{Results}

The age of patients and controls ranged from 35 till 50 years old with mean age 38.3 years old.

Regarding the smoking and dyslipidemic risk factors, we had 42 smoker patients and 30 dyslipidemic patients in total and there was no significant variation between both groups regarding these 2 factors. Age was similarly of no significant difference between both groups $(p=0.6)$. The distribution of these later risk factors and age among both groups is presented in Table 1.

The office, average and morning ambulatory blood pressure parameters were not significantly variable between both groups.

The night blood pressure parameters revealed significantly higher systolic blood pressure in the MCI group (Group 1) than in the control group (Group 2) with p value $=0.019$. The night MAP was also significantly higher in the MCI group than in the control group $(\mathrm{p}=0.01)$. This is represented in Table 2.

Echocardiographic parameters revealed significant difference between both groups regarding diastolic dysfunction ( $p=0.038$ ). MRI score was significantly different between both groups $(p=0.019)$. This is represented in Table 3.

The night systolic and mean arterial blood pressure measurements as well as the diastolic dysfunction correlated significantly to the MMSE score $(\mathrm{R}=0.49-0.6, \mathrm{p}=<0.0005-0.02)$. The night systolic and mean arterial blood pressure also correlated significantly to the MRI score $(\mathrm{R}=0.82-0.88, \mathrm{p}=0.001-0.004)$. This is represented in Table 4.

Running the linear regression test, the night mean arterial pressure and the diastolic dysfunction had the strongest impact on the MMSE class of the patient $\left(R^{2}=0.87, p=0.001\right)$. Upon the MRI score, the night systolic blood pressure had the strongest impact $\left(\mathrm{R}^{2}=0.67, \mathrm{p}=0.004\right)$.

Table 1. Comparing both groups regarding the smoking and dyslipidemic status, age and MRI score.

\begin{tabular}{cccc}
\hline & MCI Group (Group 1) & Control Group (Group 2) & Significance Level (Chi Square \& MW-U) \\
\hline Smoking & $25 / 50$ & $17 / 30$ & 0.65 \\
Dyslipidemia & $19 / 50$ & $11 / 30$ & 1.01 \\
Age & $45.5 \pm 2.6$ & $42.1 \pm 2.8$ & 0.68 \\
\hline
\end{tabular}

Table 2. Comparing both groups regarding the office and ABPM parameters.

\begin{tabular}{cccc}
\hline & MCI Group (Group 1) & Control Group (Group 2) & Significance Level (Student T-Test) \\
\hline Office Systolic BP & $135.8 \pm 5.8$ & $131.25 \pm 5.29$ & 0.3 \\
Office Diastolic BP & $81.67 \pm 7.5$ & $81.25 \pm 7.35$ & 0.46 \\
Overall ABPM Systolic BP & $114.2 \pm 6.6$ & $112.34 \pm 6.3$ & 0.4 \\
Overall ABPM Diastolic BP & $75.35 \pm 2.68$ & $68.2 \pm 2.56$ & 0.4 \\
Overall MAP & $82.33 \pm 5.56$ & $72.83 \pm 5.59$ & 0.52 \\
Awake ABPM Systolic BP & $125.3 \pm 6.15$ & $113.03 \pm 6.72$ & 0.71 \\
Awake ABPM Diastolic BP & $77.33 \pm 2.66$ & $68.38 \pm 2.15$ & 0.11 \\
Awake MAP & $82.53 \pm 3.42$ & $79.93 \pm 3.26$ & 0.4 \\
Sleep ABPM Systolic BP & $135.7 \pm 3.57$ & $112.73 \pm 2.48$ & 0.019 \\
Sleep ABPM Diastolic BP & $64.8 \pm 10.79$ & $59.8 \pm 8.96$ & 0.2 \\
Sleep MAP & $80.67 \pm 10.88$ & $70.9 \pm 7.35$ & 0.01 \\
\hline
\end{tabular}

BP = Blood Pressure. ABPM = Ambulatory Blood Pressure Monitoring. MAP = Mean Arterial Pressure. 
Table 3. Comparing both groups regarding the echocardiographic parameters and MRI score.

\begin{tabular}{cccc}
\hline & MCI Group (Group 1) & Control Group (Group 2) & $\begin{array}{c}\text { Significance Level (Chi Square, MW-U \& } \\
\text { Student T-Test) }\end{array}$ \\
\hline Left Ventricular Mass & $187.89 \pm 91.9$ & $133.15 \pm 21.11$ & 0.11 \\
Systolic Function & $63.67 \pm 8.79$ & $64.26 \pm 5$ & 0.82 \\
Diastolic Dysfunction & $38 / 50$ & $14 / 30$ & 0.015 \\
LVID & $4.64 \pm 0.98$ & $4.03 \pm 0.54$ & 0.11 \\
IVSD & $1.07 \pm 0.13$ & $1.04 \pm 0.12$ & 0.52 \\
PWD & $1.06 \pm 0.22$ & $1 \pm 0.14$ & 0.4 \\
MRI Score & $7.78 \pm 5.36$ & $1.58 \pm 1.07$ & 0.006 \\
\hline
\end{tabular}

LVID = Left Ventricular Internal Dimension in Diastole. IVSD = Inter-Ventricular Septum Dimension in Diastole. PWD = Posterior Wall Dimension in Diastole.

Table 4. Correlation between the selected parameters and each of the MMSE and MRI scores.

\begin{tabular}{ccc}
\hline & MMSE Score (R, p) & MRI Score (R, p) \\
\hline Sleep Systolic BP & $0.63,<0.0005^{*}$ & $0.58,0.001^{*}$ \\
Sleep MAP & $0.6,0.02$ & $0.58,0.001^{*}$ \\
Diastolic Dysfunction & $0.49,0.009^{*}$ & $0.32,0.095$ \\
\hline
\end{tabular}

"Correlation is significant at the 0.01 level. MAP = Mean Arterial Pressure.

\section{Discussion}

Hypertension targets many body organs, including the heart, kidneys, eyes, and brain. In apparently healthy people, hypertension can lead to mild to moderate alterations in the brain structure and function, less attention, learning and memory [14]. The hypertension-related structural and functional changes in the brain can be detected by routine brain MRI or CT scans and by neuropsychological assessment of cognitive abilities [15].

Clinic measured blood pressure is usually not representative of the patient's blood pressure throughout the day. This is the result of the several factors, e.g. white coat hypertension phenomenon, patients who are not well compliant to their anti-hypertensive treatment. Accordingly, clinicians in many centers are increasingly getting dependent on the 24-hour ABPM for the evaluation of the patient's blood pressure variation throughout the day.

In this study we aimed to evaluate the diurnal variation of blood pressure in young patients with mild cognitive impairment. Comparing the MCI group to the control group, there was no significant variation between them regarding office measured BP, average and morning ABPM parameters ( $p=0.1-0.7)$. However, sleep systolic and MAP showed significant difference between the two groups ( $\mathrm{p}=0.019$ and 0.01 respectively). We also evaluated some co-factors, like age, dyslipidemic profile and smoking status; these however were not significantly different between both groups. The male gender was selected in our sample to control this co-factor. Similarly, other co-factors, like diabetes, metabolic and psychiatric disorders, were excluded as per the study design.

The patient group was also significantly different from the control group as per the absence or presence of diastolic dysfunction $(\mathrm{p}=0.038)$ and their MRI scoring $(\mathrm{p}=0.019)$.

Bivariate correlation proved a significant strong correlation between each of the sleep systolic BP, sleep MAP and presence of diastolic dysfunction and the MMSE score $(\mathrm{R}=0.49-0.6, \mathrm{p}=<0.0005-0.02)$. For sleep systolic BP and sleep MAP, this correlation was significant at the $\mathrm{p}=0.01$ level. This agrees with results from cross-sectional [16] and longitudinal [14] [17]-[20] studies. These studies revealed a correlation between BP and cognitive function in elderly people. However, the relation of cognitive dysfunction to the systolic or diastolic $\mathrm{BP}$ is variable. Some authors reported an association between high systolic BP (The Honolulu-Asia Aging Study) and cognitive dysfunction [21]. Others reported a relation between high diastolic BP and cognitive dysfunction [19]. Other authors suggested that elevated both systolic and diastolic BP was more related to the cognitive decline [9]. 
Regarding the MRI scoring, we found that the sleep systolic BP and sleep MAP had a significant strong correlation at the $\mathrm{p}=0.01$ level with the MRI score $(\mathrm{R}=0.58, \mathrm{p}=0.001)$.

Our results revealed that the night MAP and diastolic dysfunction had the strongest impact on the MMSE score. On the other hand, the night systolic BP had the strongest impact on the cerebral MRI score. Many studies agreed with ours regarding the effect of average 24-hour and average daytime systolic blood pressure to the cognitive decline and MRI changes [9] [10]. Nagai M et al., however disagreed in that the sleeping systolic BP had the stronger impact upon the cognitive ability, although average overall and average awake systolic BP were also strongly correlated to cognitive function [22]. To the authors' best knowledge, few researchers have found a strong correlation between the cognitive impairment and the diastolic blood pressure at any phase of the 24-hour ABPM [19] [23].

Silent cerebral white matter lesions (WMLs) represent an important prognostic factor as they are considered a risk factor for the development of stroke, dementia and cognitive impairment. This is true for the elderly, where these silent WMLs are a common finding on brain magnetic resonance imaging (MRI). The pathogenesis of these WMLs is debatable, but aging and hypertension are reported to be the main risk factors [24] [25]. However, in young age cerebral WMLs are not a common finding in brain MRI.

In hypertensive patients, WMLs tend to occur earlier in life and appear more extensive, compared to normotensive individuals [24] [26]. In addition, it has been shown that treated controlled hypertensive patients have a lower prevalence of WML than both untreated and treated but not controlled hypertensive patients [26].

Our study had some drawbacks. First, we did not include diabetic patients. We aimed to make the study sample as uniform as possible, accordingly we excluded diabetes as an important risk factor for vasculopathy and hypertension. This implies that our results are only applicable to non-diabetic hypertensive patients.

Second, some other factors could not be excluded from the study, for example the dyslipidemia and smoking factors. These were however insignificant among the 4 groups of patients. Age is also an important risk factor for altered cognitive function and hypertension. In our sample, the severe cognitive impairment group had a higher mean age that reached a weak statistical significance level $(\mathrm{p}=0.03)$. This is possibly due to a higher minimum age in that group (70 years old vs 65 years old for the other 3 groups). Also, this poor significance does not explain the association with uncontrolled hypertension seen in the mild and moderate cognitive impairment groups. Hypertension duration is also among the strongly significant factors that affected the patients' cognitive ability and their cerebral MRI scores. This seems to be a logical relation and based on the multiple regression analysis, age and hypertension duration had a lower impact on the MMSE and cerebral MRI score than the average awake systolic blood pressure.

Third, the effect of treatment and the achievement of a well-controlled BP were not evaluated in our study. This is a very important issue that needs a longitudinal study with long term follow up to record the rate of change of cognitive decline and cerebral MRI score, not to mention studying the different effects of variable drug combinations that achieve the proper BP control.

Finally, the time duration of poor control of hypertension until the appearance of cognitive impairment is a factor that needs a longitudinal study to record its impact upon the utility of MMSE test for the prediction of hypertension control. But this point may be of little importance as the authors do not suggest the replacement of 24-hour ABPM by the clinic MMSE test.

\section{Conclusion}

We conclude from our study that MMSE is a simple test to run in clinic and can give an idea about the degree of structural damage caused to the brain by hypertension; and hence predict whether or not the patient's BP is well controlled. This will not replace the 24-hour ABPM for the actual identification of the degree of BP control but the abnormal MMSE score may help guide the clinicians to request this 24-hour monitoring. 24-hour ABPM is still an essential tool for ongoing evaluation of patients' BP and following up the effect of treatment. Also MCI in normotensive young adult patients can reflect an abnormal circadian blood pressure rhythm. Ambulatory blood pressure monitoring can be an essential investigation in young adult MCI patients.

\section{Conflicts of Interest}

The authors report no conflicts of interest. 


\section{References}

[1] Pickering, T. (1996) Recommendations for the Use of Home (Self) and ABPM Monitoring. American Journal of Hypertension, 9, 1-11. http://dx.doi.org/10.1016/0895-7061(95)00341-X

[2] Giuseppe, M., Staessen, J.A., Gasowski, J., et al. (2007) The Task Force for the Management of Arterial Hypertension of the European Society of Hypertension (ESH) and of the European Society of Cardiology (ESC). European Heart Journal, 28, 1462-1536.

[3] Sheps, S.G., Clement, D.L., Pickering, T.G., et al. (1994) Ambulatory Blood Pressure Monitoring. Hypertensive Diseases Committee, American College of Cardiology. Journal of the American College of Cardiology, 23, 1511-1513. http://dx.doi.org/10.1016/0735-1097(94)90400-6

[4] Folstein, M.F., Folstein, S.E. and McHugh, P.R. (1975) Mini-Mental State. A Practical Method for Grading the Cognitive State of Patients for the Clinician. Journal of Psychiatric Research, 12, 189-198. http://dx.doi.org/10.1016/0022-3956(75)90026-6

[5] Wrobel, N.H. and Farrag, M.F. (2007) Preliminary Validation of an Arabic Version of the MMSE in the Elderly. Clinical Gerontologist, 31, 75-93. http://dx.doi.org/10.1080/07317110802072223

[6] Crum, R.M., Anthony, J.C., Bassett, S.S. and Folstein, M.F. (1993) Population-Based Norms for the Mini-Mental State Examination by Age and Educational Level. The Journal of the American Medical Association, 269, 2386-2391. http://dx.doi.org/10.1001/jama.1993.03500180078038

[7] Scheltens, P., Barkhof. F., Leys, D., et al. (1993) A Semiquantitative Rating Scale for the Assessment of Signal Hyperintensities on Magnetic Resonance Imaging. Journal of the Neurological Sciences, 114, 7-12. http://dx.doi.org/10.1001/jama.1993.03500180078038

[8] Faul, F., Erdfelder, E., Buchner, A. and Lang, A.G. (2009) Statistical Power Analyses Using G*Power 3.1: Tests for Correlation and Regression Analyses. Behavior Research Methods, 41, 1149-1160. http://dx.doi.org/10.3758/BRM.41.4.1149

[9] van Boxtel, M.P.J., Henskens, L.H.G., Kroon, A.A., Hofman, P.A.M., Gronenschild, E.H.B.M., Jolles, J. and de Leeuw, P.W. (2006) Ambulatory Blood Pressure, Asymptomatic Cerebrovascular Damage and Cognitive Function in Essential Hypertension. Journal of Human Hypertension, 20, 5-13. http://dx.doi.org/10.1038/sj.jhh.1001934

[10] Hayakawa, M., Yano, Y., Kuroki, K., Inoue, R., Nakanishi, C., Sagara, S., et al. (2012) Independent Association of Cognitive Dysfunction with Cardiac Hypertrophy Irrespective of 24-h or Sleep Blood Pressure in Older Hypertensives. American Journal of Hypertension, 25, 657-663. http://dx.doi.org/10.1038/ajh.2012.27

[11] White, W.B., Wolfson, L., Wakefield, D.B., Hall, C.B., Campbell, P., Moscufo, N., et al. (2011) Average Daily Blood Pressure, Not Office Blood Pressure, Is Associated With Progression of Cerebrovascular Disease and Cognitive Decline in Older People. Circulation, 124, 2312-2319. http://dx.doi.org/10.1161/CIRCULATIONAHA.111.037036

[12] Shimada, K., Kawamoto, A., Matsubayashi, K., Nishinaga, M., Kimura, S. and Ozawa, T. (1992) Diurnal Blood Pressure Variations and Silent Cerebrovascular Damage in Elderly Patients with Hypertension. Journal of Hypertension, 10, 875-878.

[13] Goldstein, I.B., Bartzokis, G., Hance, D.B. and Shapiro, D. (1998) Relationship between Blood Pressure and Subcortical Lesions in Healthy Elderly People. Stroke, 29, 765-772. http://dx.doi.org/10.1161/01.STR.29.4.765

[14] Elias, M.F., Wolf, P.A., D’Agostino, R.B., et al. (1993) Untreated Blood Pressure Level Is Inversely Related to Cognitive Functioning: The Framingham Study. American Journal of Epidemiology, 138, 353-364.

[15] Waldstein, S.R., Brown, J.R., Maier, K.J. and Katzel, L.I. (2005) Diagnosis of Hypertension and High Blood Pressure Levels Negatively Affect Cognitive Function in Older Adults. Annals of Behavioral Medicine, 29, 174-180. http://dx.doi.org/10.1207/s15324796abm2903 3

[16] Cacciatore, F., Abete, P., Ferrara, N., Paolisso, G., Amato, L., Canonico, S., et al. (1997) The Role of Blood Pressure in Cognitive Impairment in an Elderly Population. Journal of Hypertension, 15, 135-142. http://dx.doi.org/10.1097/00004872-199715020-00003

[17] Dufouil, C., de Kersaint-Gilly, A., Besancon, V., Levy, C., Auffray, E., Brunnereau, L., et al. (2001) Longitudinal Study of Blood Pressure and White Matter Hyperintensities: The EVA MRI Cohort. Neurology, 56, 921-926. http://dx.doi.org/10.1212/WNL.56.7.921

[18] de Leeuw, F.E., de Groot, J.C., Oudkerk, M., Witteman, J.C., Hofman, A., van Gijn, J. and Breteler, M.M. (2012) Hypertension and Cerebral White Matter Lesions in a Prospective Cohort Study. Brain, 125, 765-772. http://dx.doi.org/10.1093/brain/awf077

[19] Kilander, L., Nyman, H., Boberg, M., Hansson, L. and Lithell, H. (1998) Hypertension Is Related to Cognitive Impairment: A 20-Year Follow-Up of 999 Men. Hypertension, 31, 780-786. http://dx.doi.org/10.1161/01.HYP.31.3.780

[20] Carmelli, D., Swan, G.E., Reed, T., Miller, B., Wolf, P.A., Jarvik, G.P. and Schellenberg, G.D. (1998) Midlife Cardiovascular Risk Factors, ApoE, and Cognitive Decline in Elderly Male Twins. Neurology, 50, 1580-1585. 
http://dx.doi.org/10.1212/WNL.50.6.1580

[21] Havlik, R.J., Foley, D.J., Sayer, B., Masaki, K., White, L. and Launer, L.J. (2002) Variability in Midlife Systolic Blood Pressure Is Related to Late-Life Brain White Matter Lesions: The Honolulu-Asia Aging Study. Stroke, 33, 26-30. http://dx.doi.org/10.1161/hs0102.101890

[22] Nagai, M., Hoshide, S., Ishikawa, J., Shimada, K. and Kario, K. (2008) Ambulatory Blood Pressure as an Independent Determinant of Brain Atrophy and Cognitive Function in Elderly Hypertension. Journal of Hypertension, 26, 16361641. http://dx.doi.org/10.1097/HJH.0b013e3283018333

[23] Prybyl's'ka, O.S. (2010) Predictors of Cognitive Impairment in Patients with Essential Hypertension. Likars'ka Sprava, 28-33.

[24] Pantoni, L. and Garcia, J.H. (1995) The Significance of Cerebral White Matter Abnormalities 100 Years after Binswanger's Report: A Review. Stroke, 26, 1293-1301. http://dx.doi.org/10.1161/01.STR.26.7.1293

[25] Pantoni, L. (2010) Cerebral Small Vessel Disease: From Pathogenesis and Clinical Characteristics to Therapeutic Challenges. The Lancet Neurology, 9, 689-701. http://dx.doi.org/10.1016/S1474-4422(10)70104-6

[26] Liao, D., Cooper, L., Cai, J., Toole, J.F., Bryan, N.R., Hutchinson, R.G. and Tyroler, H.A. (1996) Presence and Severity of Cerebral White Matter Lesions and Hypertension, Its Treatment, and Its Control: The ARIC Study. Stroke, 27, $2262-$ 2270. http://dx.doi.org/10.1161/01.STR.27.12.2262 